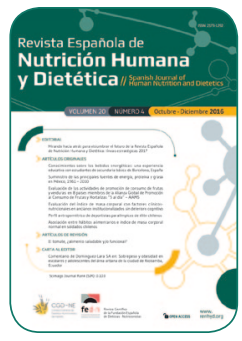

www.renhyd.org

\title{
EDITORIAL
}

\section{Mirando hacia atrás para vislumbrar el futuro de la Revista Española de Nutrición Humana y Dietética: líneas estratégicas 2017}

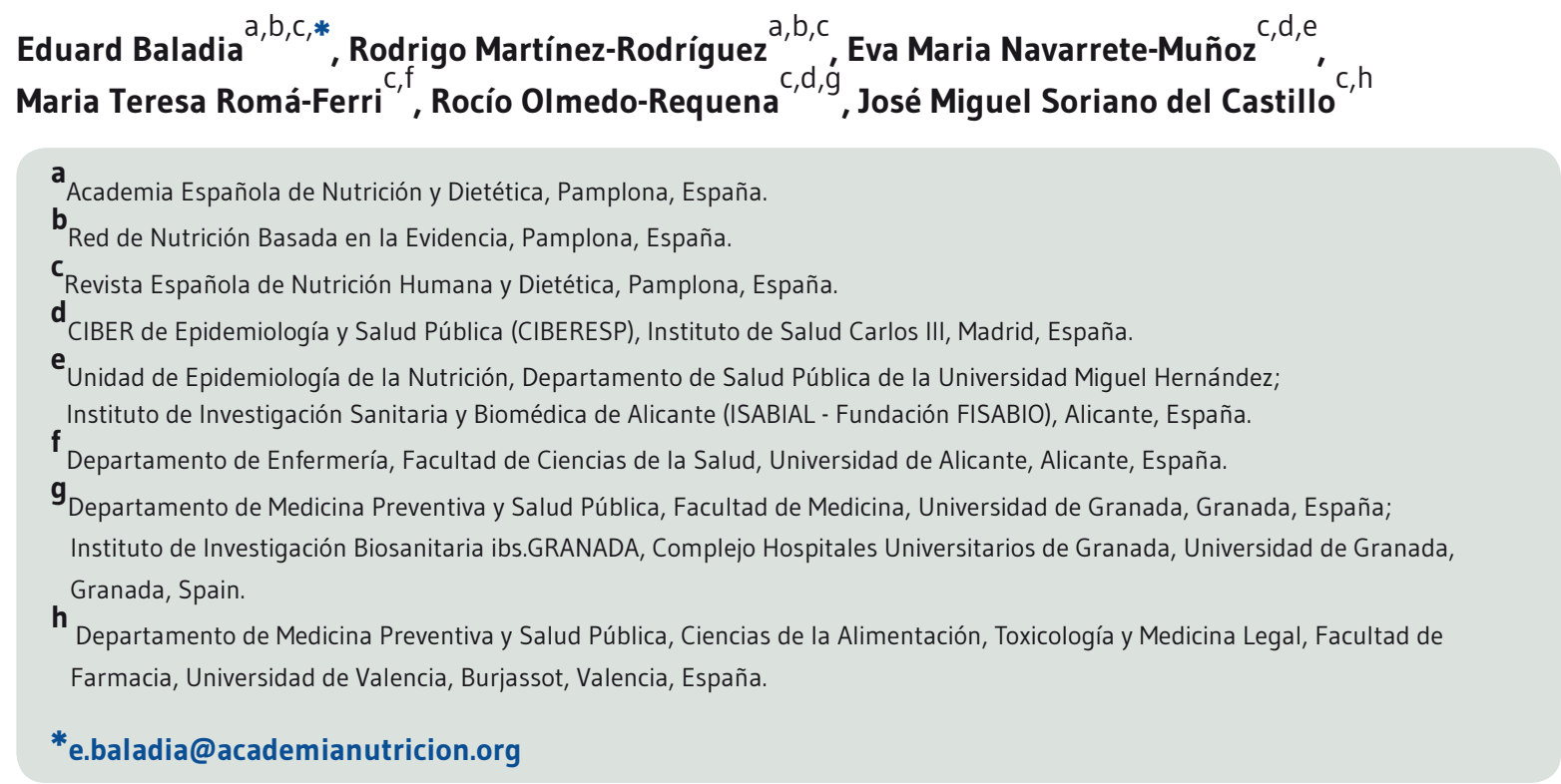

Recidido el 17 de diciembre de 2016; aceptado el 18 de diciembre de 2016.

\section{CITA}

Baladia E, Martínez-Rodríguez R, Navarrete-Muñoz EM, Romá-Ferri MT, Olmedo-Requena R, Soriano del Castillo JM. Mirando hacia atrás para vislumbrar el futuro de la Revista Española de Nutrición Humana y Dietética: líneas estratégicas 2017. Rev Esp Nutr Hum Diet. 2016; 20(4): 258-62. doi: 10.14306/renhyd.20.4.341

“Empezar de cero nos da la oportunidad de pensar en el pasado, sopesar las cosas que hemos hecho y aplicar lo que hemos aprendido de esas experiencias en el futuro"1. Y es que cada año la Revista Española de Nutrición Humana y Dietética (RENHyD) tiene la oportunidad, y la obligación, de adaptarse a los nuevos requisitos de la difusión científica internacional, con el propósito siempre de mejorar. El 8 de octubre de 2016 se reunió en Alicante el Comité Editorial de la revista para echar la vista atrás y poder delimitar las líneas estratégicas prioritarias para 2017.
Desde 2013 se ha conseguido, entre otros, que la Revista Española de Nutrición Humana y Dietética sea una revista científica sin publicidad y financiada íntegramente por la Academia Española de Nutrición y Dietética (anteriormente Fundación Española de Dietistas-Nutricionistas), completamente autogestionada por el equipo editorial, de acceso abierto y sin coste económico para las/los autoras/es. A la vista de los grandes conflictos de interés financieros que existen en el campo de la nutrición humana y dietética ${ }^{2,3}$, uno de los propósitos en los que seguiremos trabajando 
en los próximos años es conseguir que sea una revista que cuente con la declaración pública de forma obligatoria de los conflictos de intereses tanto del equipo editorial como de los/as revisores/as y de los/as autores/as. En definitiva, es la comunidad de Dietistas-Nutricionistas la que está permitiendo que se publiquen las investigaciones que se realizan en el campo de la Nutrición Humana y Dietética de todo el mundo, lo que contribuye a consolidar su campo de conocimiento, a innovar y a garantizar las pruebas imparciales que permitan el desarrollo de competencias más ajustadas a los cambios sociales y a las demandas de la población.

La decisión de generar una revista sólo en formato electrónico y de autogestionar todo el proceso editorial, desde la gestión de artículos (incluyendo todas las fases de revisión por pares), gestión de metadatos (generación y registro para obtención de DOIs), gestión de página web (incluyendo el sistema de gestión de manuscritos), registro en bases de datos y actualización, ha permitido que la revista pueda crecer en producción científica y calidad sin poner en riesgo su sostenibilidad económica. El esfuerzo humano del equipo editorial, el entendimiento y buen hacer de unas/os socias/os que han dejado de recibir la revista en formato papel para obtener un nuevo formato que facilita su consulta en cualquier lugar y en el momento que lo precisen, y el apoyo incondicional de los patronos de la Academia Española de Nutrición y Dietética y de todas/os las/los presidentas/es de Asociaciones y Colegios de Dietistas-Nutricionistas, son los principales agentes que lo han hecho posible. A todos ellos, gracias.

En estos años la revista ha conseguido ser indexada en: SCOPUS, EMBASE/Excerpta Medica, DOAJ (Directory of Open Access Journals), REDIB (Red Iberoamericana de Innovación y Conocimiento Científico), Dialnet, WorldCat (World Catalog OCLC), el catálogo colectivo REBIUN (Red de Bibliotecas Universitarias Españolas), lo que ha incrementado su visibilidad y ha aumentado la posibilidad de ser consultada y empleada para apoyar nuevos trabajos científicos. La indexación que ha propiciado la publicación de un mayor número de artículos de investigación tanto por autores nacionales como internacionales, ha sido la incorporación de RENHyD a SciELO (Scientific Electronic Library Online), una de las colecciones de revistas científicas de ciencias de la salud más importantes a nivel latinoamericano. El Comité Editorial está seguro de que, además, este proceso de edición y de visualización ha influido en lograr la incorporación en un nuevo recurso de Thomson Reuters como Emerging Source de Citation Index ${ }^{4}$.

Aunque está bien celebrar el éxito, es más importante prestar atención a las lecciones aprendidas durante este recorrido. Con los resultados positivos también se encuentran los resultados no conseguidos. Estos últimos son una forma objetiva de conocer lo que nos falta por lograr y una directriz de lo que se puede mejorar y cómo intentar alcanzar un prestigio como medio de comunicación científico. En 2012 la revista se presentó para ser indexada en PubMed, consiguiendo una puntuación global de 3 en una escala de Likert de 0 a 5 (Figura 1), en el año 2014 se solicitó la evaluación por Thomson Reuters y en el año 2015 la revista se presentó para ser indexada en MEDLINE (Figura 2). Tres veces rechazados y tres pistas para mejorar: aumentar la cantidad de artículos de investigación publicados, aumentar la cantidad de veces que otras revistas citan RENHyD y aumentar la calidad de las investigaciones publicadas, ampliando la diversidad de los diseños, e incrementar los tamaños y la representatividad de las muestras de población estudiadas.

Figura 1. Resultados de la evaluación de la Revista Española de Nutrición Humana y Dietética para ser indexada en PubMed.
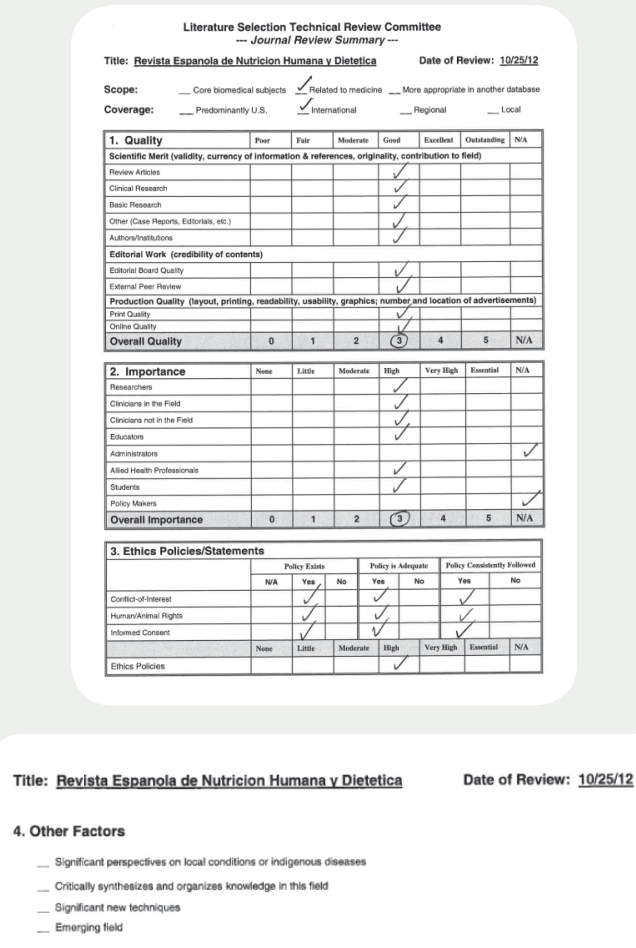

Journal Strengths: The editorial board membership is geographically diverse and includes members from Spain, Chile, Penu, Argentina, Brazil, Costa Rica, tialy and the US. Abstracts are structured. $90 \%$ of Consent, and Human/Animal Rights. English-language articies reviewed that involved human subjects included clear statements about Informed Consent and Ethical board review in their methods sections. The website was easy to navigate and included both full-text HTML and PDF versions of articles.

Area(s) for Improvement: Studies are mostly observational with small sample sizes.

Overall Comments: This is a journal established in 1997 that focuses on nutrition and diet. It is publishe by the Spanish Association of Diettilans and Nutritionists. Arricles are mostly in Spanish but a few in each

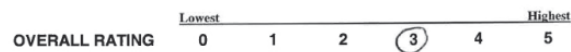


Figura 2. Respuestas de Thomson Reuters y de MEDLINE sobre la propuesta de la Revista Española de Nutrición Humana y Dietética para ser evaluada de su factor de impacto e indexada en MEDLINE, respectivamente.
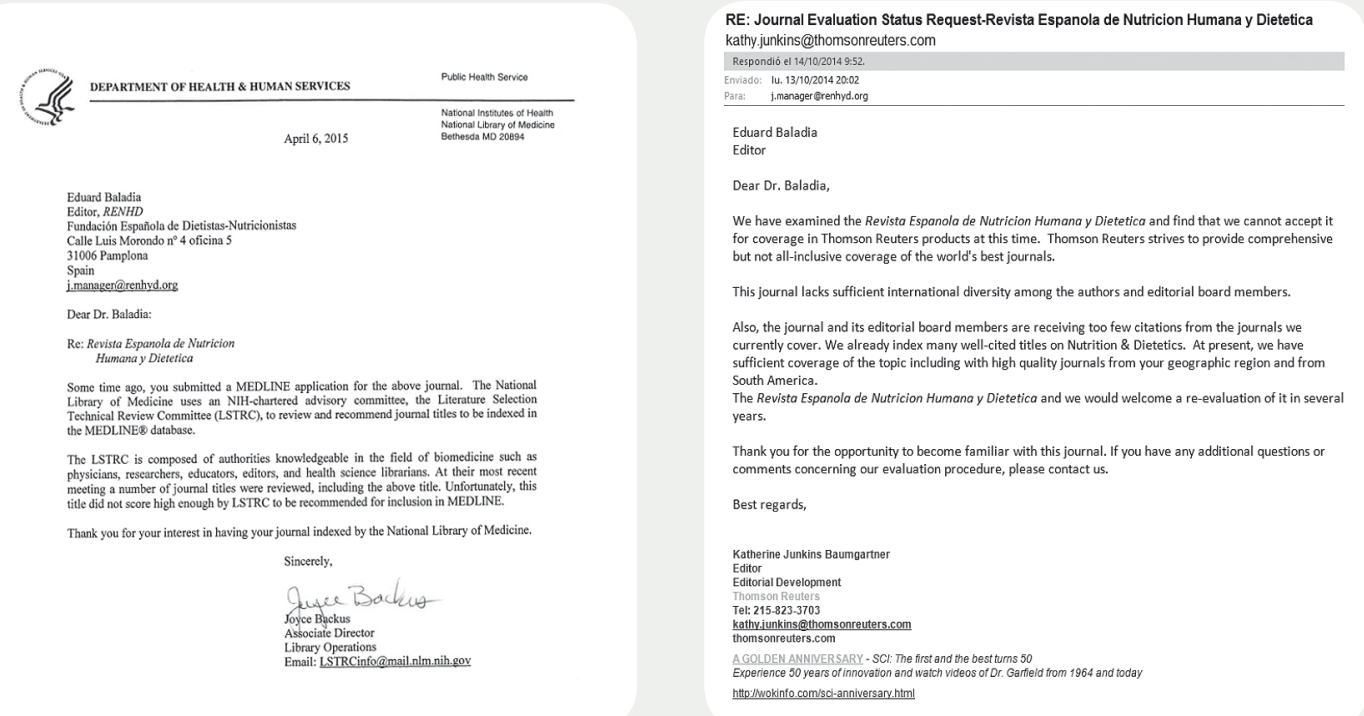

"Incluso un camino sinuoso difícil, nos puede conducir a la meta si no lo abandonamos hasta el final"5. El aumento de publicación de artículos de investigación en números absolutos y en proporción al resto de artículos es más que notorio. En 2013 se publicaban un total de 22 artículos, de los cuales sólo el $36,4 \%$ eran artículos de investigación $(n=8)$, y el resto eran de revisión o similares $(n=14)$. En 2016, se han editado 36 artículos, de los cuales más del $70 \%$ son artículos de investigación $(n=26)$, y el resto son artículos de revisión o similares $(n=10)$. En un cuatrienio se ha logrado triplicar la publicación de artículos de investigación (ver la evolución del tipo de artículos publicados entre 2013 y 2016 en la Figura 3). La comparación de datos con ciclos anteriores de la revista es aún más Ilamativa. En estos 4 últimos años se ha publicado casi la misma cantidad de artículos que en los 10 años iniciales (1997-2007), y 4 veces más artículos de investigación, superando la previsión inicial de crecimiento ${ }^{6}$.

Y es que la revista no sólo ha conseguido un crecimiento del $34 \%$ en tan sólo 4 años en la publicación de artículos, sino que el número de artículos recibidos también ha aumentado considerablemente de 2013 a 2016, lo que ha conllevado a aumentar las exigencias de calidad metodológica de los mismos, consiguiéndose con ello mejorar, y a fomentar la publicación de artículos originales con el fin de alcanzar los estándares internacionales de proporción de artículos de investigación necesaria para que la revista mejore su evaluación de calidad formal como medio de difusión científico, lo cual permite cumplir con uno de los requisitos básicos de las actuales bases de datos documentales. Las/ los autoras/es que han decidido publicar en RENHyD son el cuerpo humano y académico que ha permitido tal logro. Muchas gracias, sin vosotras/os, la revista no podría existir.

Figura 3. Evolución de la cantidad de artículos de investigación publicados en la Revista Española de Nutrición Humana y Dietética entre 2013 y 2016.

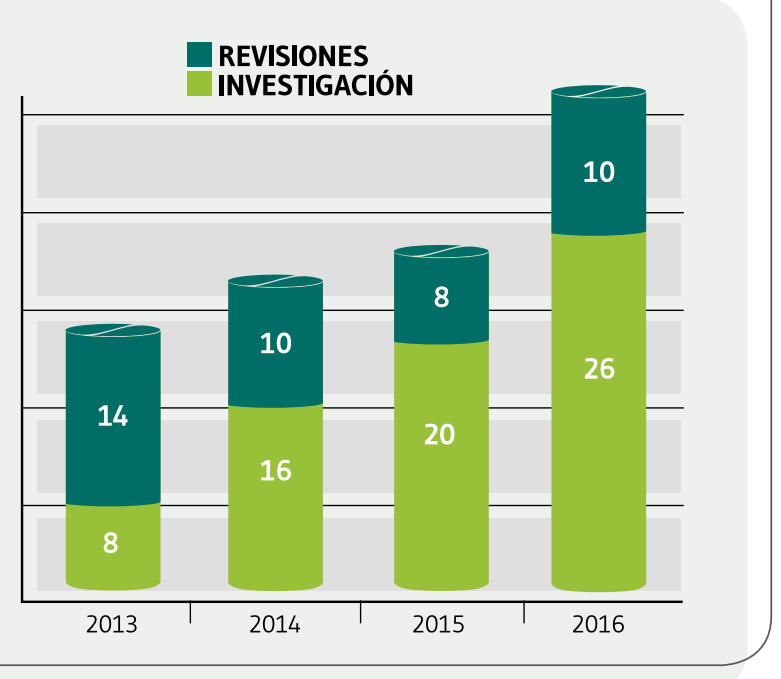


"Hay miserables afanes de popularidad, más denigrantes que el servilismo"7. El aumento de la publicación de más artículos junto con el efecto del acceso abierto, hace que actualmente la revista sea mucho más visible en todo el mundo, lo que permitirá aumentar la probabilidad de la citación de sus artículos. Respecto a la influencia lograda a través de la página web de la revista, en 2016 se recibieron más de 36.000 usuarios (5.000 usuarios más que en 2015) que ejecutaron más de 50.000 sesiones de lectura o descarga de artículos publicados (un $26 \%$ más que en 2015). La procedencia de los usuarios, de acuerdo a la IP de su acceso a Internet, fue principalmente de España (43\%), México (18\%), Chile, Colombia, Ecuador y Perú (con $5 \%$ de cuota cada uno), Argentina (4\%), Estados Unidos, Venezuela y Brasil (con un $1,6 \%$ de cuota cada uno). A pesar del éxito potencial de la de influencia lograda mediante del aumento de la visibilidad de la revista a través de la página web, la influencia de una revista es medida objetivamente por el número de citas que logran los artículos publicados en un período de tiempo determinado (en los dos o tres años siguientes). Uno de los indicadores de dicho impacto es el que proporciona Scimago Journal \& Country Rank (SJR) 8 que asocia tanto las citas recibidas como la importancia que tiene la propia revista en la que se cita. El valor de impacto de este último trienio ha sido variable y ha oscilado entre 0.156 (2013), 0.122 (2014) y 0.125 (2015). No obstante, si consultamos los datos absolutos de citas externas recibidas nos encontramos con una evolución positiva (Tabla 1), habiendo pasado de obtener 8 citas en revistas externas a 14 citas. Aunque se está logrando una repercusión ascendente, no es suficiente para destacar entre todas las revistas publicadas cuya cobertura temática es la Nutrición Humana y la Dietética. La colección de revistas de esta temática en el SJR es de 115 y el puesto ocupado ha pasado del 90 en 2013 al puesto 104 en 2015; asimismo la colección incluida en el Journal Citation Reports ${ }^{\circledR}$ en 2015 está compuesta por 80 revistas con amplia influencia y reconocimiento internacional. En este sentido, la Revista Española de Nutrición Humana y Dietética tiene todavía mucho camino por recorrer, y entre todos debemos valorar críticamente estos datos para seguir mejorándola e

Tabla 1. Evolución de citas de RENHyD en cifras absolutas por años.

\begin{tabular}{l|c|c} 
Año & Citas totales & Autocitas*/Externas \\
\hline 2013 & 17 & $9 / 8$ \\
2014 & 10 & $4 / 6$ \\
2015 & 16 & $2 / 14$
\end{tabular}

* Autocitas alude a cuando se cita un artículo publicado en la propia revista. incrementando la repercusión de la producción científica que a través de ella se realiza para generar nuevo conocimiento en nuestra disciplina.

A pesar de las cifras obtenidas, el Comité Editorial desea agradecer a las/los autoras/es y lectoras/es su apoyo incondicional, al fin y al cabo, sin consumidores de información la revista no sirve a la comunidad científica.

Viendo los avances realizados desde las últimas evaluaciones recibidas y los asuntos que han quedado pendientes, en la reunión del 8 de octubre de 2016 se vislumbraron las siguientes líneas estratégicas con el fin de posibilitar en un futuro cercano la indexación en MEDLINE y cumplir los criterios para poder entrar en las bases de datos Science Citation Index (SCI), Social Science Citation Index (SSCI) y JCR producidas por Thomson Reuters:

- Favorecer la publicación en inglés sin descuidar la publicación hispano-hablante, de la que, en parte, somos responsables en el terreno de la Nutrición Humana y Dietética. El Comité Editorial entiende que esto favorecerá que la revista sea accesible a un mayor número de usuarias/os.

- Priorizar la publicación de artículos originales de investigación y metaanálisis.

- Aumentar la calidad metodológica de los artículos publicados. A este respecto, se han incorporado y se incorporarán nuevas/os editoras/es con experiencia en metodología y estadística aplicada a la investigación y se vigilará especialmente que la representatividad de la muestra, el uso de herramientas validadas y la reproducibilidad de la investigación junto a una concordancia entre el análisis estadístico y el objeto del estudio sea abordado por las/os autoras/es de forma adecuada.

- Priorizar la publicación de manuscritos que sean de relevancia para la Dietética y Nutrición y que aporten una visión novedosa de su investigación.

- Permitir la publicación de protocolos de investigación de proyectos que se estén llevando a cabo en el campo de la Nutrición y la Dietética, dando preferencia a aquellos que cuenten con financiación de una convocatoria abierta y competitiva.

- Revisar el Consejo Editorial y la base de datos de Revisoras/es externos para agilizar los trámites de revisión por pares y mejorar los tiempos de respuesta de la revisión de manuscritos. 
- Redacción de unas nuevas normas de publicación para autores/as y documentos de ayuda para editores/as y revisores/as con las políticas del proceso editorial.

- Promover la colaboración activa entre revistas hispanohablantes para obtener la excelencia en estándares de publicación.

- Programar sesiones periódicas de trabajo del Comité Editorial con el fin de mejorar el proceso de publicación de manuscritos de la RENHyD y poder agilizar los procesos de publicación.

Vistas y aprobadas las líneas estratégicas, cabe recordar que al final, lo que importa no son los años de vida de RENHyD, sino la vida de estos años en la revista.

¡Gracias a tod@s!

Comité Editorial de la Revista Española de Nutrición Humana y Dietética.

\section{REFERENCIAS}

(1) Palacio RJ. Wonder. August y yo: Tres historias de Wonder. España: Penguin Random House Grupo Editorial España; 2016.

(2) Baladia E, Martínez-Rodríguez R. Conflictos de interés en nutrición humana y dietética. Rev Esp Nutr Hum Diet. 2016; 20(2): 77-79. doi: 10.14306/renhyd.20.2.261

(3) Chartres N, Fabbri A, Bero LA. Association of Industry Sponsorship With Outcomes of Nutrition Studies: A Systematic Review and Meta-analysis. JAMA InternMed. 2016 Dec 1; 176(12): 1769-1777. doi: 10.1001/jamainternmed.2016.6721

(4) Martínez-Rodríguez R, Baladia E, Romá-Ferri MT, MarquesLopes I. Publicar en otras revistas citando a la Revista Española de Nutrición Humana y Dietética puede ayudar a conseguir el Impact Factor: Thomson Reuters ${ }^{\circledR}$ nos observa. Rev Esp Nutr Hum Diet. 2016; 20(1): 1-3 . DOI: 10.14306/renhyd.20.1.232

(5) Cohelo P. Maktub. España: Editorial Planeta SA; 2002.

(6) Baladia E, Basulto J, Viózquez M, Cantós D. Análisis de la producción científica de ACTIVIDAD DIETÉTICA (1997-2007): ¿qué ha sido, qué es y qué se espera que sea la revista científica de la AED-N?. Rev Esp Nutr Hum Diet. 2008; 12: 5-10.

(7) Ingenieros J. El hombre mediocre. Linkgua digital; 2014.

(8) Scimago Journal \& Country Rank (SJR). Revista Española de Nutrcición Humana y Dietética [base de datos en Internet]. SCImago: Consejo Superior de Investigaciones Científicas (CSIC), Universidad de Granada, Universidad de Extremadura, Universidad Carlos III (Madrid), Universidad de Alcalá de Henares; 2016. Disponible en: http://www.scimagojr.com/ journalsearch.php?q=20100195029\&tip=sid\&clean $=0$ 